\title{
Graphene mode-locked femtosecond Alexandrite laser
}

\author{
Can Cihan, ${ }^{1}$ Coskun Kocabas, ${ }^{2}$ Umit Demirbas, ${ }^{3}$ (1) and Alphan Sennaroglu $1,4, *$ \\ ${ }^{1}$ Laser Research Laboratory, Departments of Physics and Electrical-Electronics Engineering, Koç University, Istanbul 34450, Turkey \\ ${ }^{2}$ Department of Physics, Bilkent University, Ankara 06800, Turkey \\ ${ }^{3}$ Laser Technology Laboratory, Department of Electrical and Electronics Engineering, Antalya Bilim University, 07190 Dosemealti, Antalya, Turkey \\ ${ }^{4}$ Koc University Surface Science and Technology Center (KUYTAM), Rumelifeneri, Istanbul 34450, Turkey \\ *Corresponding author: asennar@ku.edu.tr
}

Received 2 July 2018; accepted 11 July 2018; posted 23 July 2018 (Doc. ID 336531); published 9 August 2018

\begin{abstract}
We report for the first time, to the best of our knowledge, graphene mode-locked operation of a femtosecond Alexandrite laser at $750 \mathrm{~nm}$. A multipass-cavity configuration was employed to scale the output energy and to eliminate spectral/Q-switching instabilities. By using a monolayer graphene saturable absorber, mode locking could be obtained. With $5 \mathrm{~W}$ of pump at $532 \mathrm{~nm}$, nearly transformlimited, $65 \mathrm{fs}$ pulses with a time-bandwidth product of 0.319 were generated. The mode-locked laser operated at a pulse repetition rate of $5.56 \mathrm{MHz}$ and produced $8 \mathrm{~mW}$ output power, corresponding to a pulse energy and peak power of $1.4 \mathrm{~nJ}$ and $22 \mathrm{~kW}$, respectively. These experiments further show that graphene can be used to initiate mode locking at wavelengths as low as $750 \mathrm{~nm}$. () 2018 Optical Society of America
\end{abstract}

OCIS codes: (140.3580) Lasers, solid-state; (140.4050) Mode-locked lasers; (140.7090) Ultrafast lasers; (140.5680) Rare earth and transition metal solid-state lasers.

https://doi.org/10.1364/OL.43.003969

Graphene possesses a unique ultrabroad saturable absorption band with picosecond relaxation, making it possible to initiate pulsed operation of a wide range of lasers [1,2]. In particular, graphene and graphene-based supercapacitor structures have been utilized as saturable absorbers to generate $Q$-switched or mode-locked pulses from lasers operating between 603

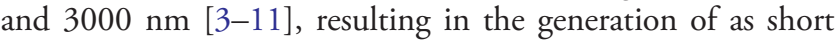
as $19 \mathrm{fs}$ pulses [7].

Recently, there has also been a renewed interest in the development of Alexandrite $\left(\mathrm{Cr}^{3+}: \mathrm{BeAl}_{2} \mathrm{O}_{4}\right)$ lasers, following the emergence of efficient diode or solid-state pump lasers in the visible. To date, efficient pulsed and continuous-wave regimes of operation have been demonstrated [12-17]. Furthermore, by utilizing the broad tuning range of the Alexandrite gain medium, several recent studies have focused on ultrashort pulse generation. In particular, Ghanbari et al. reported the generation of femtosecond pulses from a Kerr-lens mode-locked (KLM) Alexandrite laser for the first time [18]. The same group later succeeded in producing $380 \mathrm{fs}$ pulses at $775 \mathrm{~nm}$ by using an $\mathrm{InP} / \mathrm{InGaP}$ quantum dot semiconductor saturable absorber mirror [19]. The shortest pulses of $70 \mathrm{fs}$ duration were obtained from a multipass-cavity (MPC) KLM Alexandrite laser [20].

Several alternative saturable absorbers have been used at wavelengths below $800 \mathrm{~nm}$, including graphene oxide [21], semiconductor saturable absorber mirrors (SESAMs) [22], single-walled carbon nanotubes [23], quantum dots (QDs) $[19,24,25]$, and emerging two-dimensional (2D) materials [26-28]. Two-dimensional materials such as transition metaldoped dichalcogenides, black phosphorus, and topological insulators can provide strong absorption saturation but may also introduce a high level of nonsaturable loss [29-31], which is undesirable in a low-gain laser medium such as Alexandrite. Furthermore, resonant structures such as SESAMs or QDs typically operate over a relatively narrow spectral window, limiting the operational range of mode-locked lasers. In the case of graphene, its use as a saturable absorber has been scarce at wavelengths below $800 \mathrm{~nm}$ and mainly limited to $Q$-switching $[3,4]$. Mode-locking applications of graphene in this spectral range appear more challenging due to the increase of the saturation intensity with decreasing wavelength $[6,8,32,33]$. Hence, a MPC architecture or high-power pumping can be used to increase the intracavity intensities to obtain sufficient absorption saturation for the initiation of mode locking.

In this Letter, we describe, to the best of our knowledge, the first use of a graphene saturable absorber (GSA) for the mode locking of an Alexandrite laser. In the experiments, a MPC extension was added to the short Alexandrite cavity to scale up the pulse energy and also to eliminate the spectral/ $Q$-switching instabilities, which were observed in short-length resonators $[20,34]$. Pulses with a duration of 65 fs were generated near $750 \mathrm{~nm}$ with $5 \mathrm{~W}$ of pump power. These results further show that graphene can be used as a mode locker at wavelengths as short as $750 \mathrm{~nm}$.

To demonstrate graphene mode locking of the Alexandrite laser, we adopt a resonator configuration similar to what was previously used for KLM experiments [20]. Figure 1 shows a schematic of the experimental setup. Initially, a folded $x$-cavity was built by using four flat (M3-M6) and two curved (M1 and M2, $R=75 \mathrm{~mm}$ ) high reflectors, and a flat output 


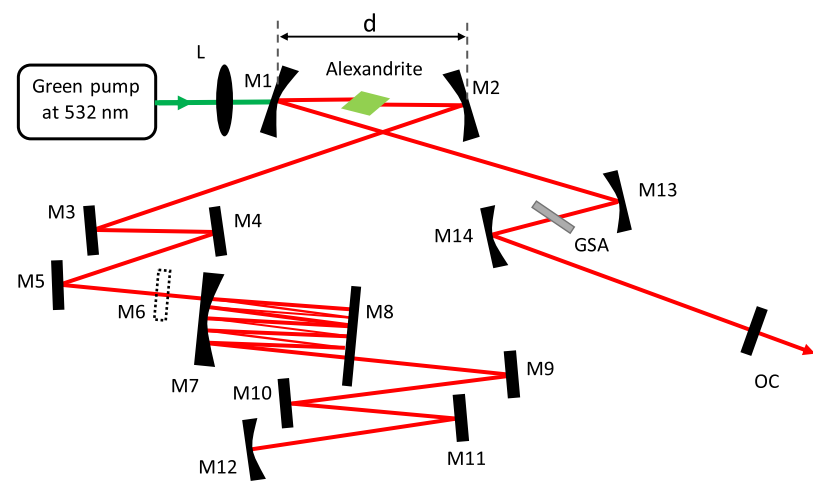

Fig. 1. Schematic of the 532-nm pumped, multipass-cavity femtosecond Alexandrite laser, mode locked near $750 \mathrm{~nm}$ with a monolayer graphene saturable absorber.

coupler (OC). Then, two curved high reflectors (M13-M14, $R=75 \mathrm{~mm}$ ) were positioned to create an additional intracavity waist at the location of the GSA. The monolayer graphene sample used in this work was grown on an infrasil substrate by using chemical vapor deposition. The fast and slow decay times of the GSA were previously measured to be $128 \mathrm{fs}$ and $1.6 \mathrm{ps}$, respectively [35]. No active cooling of the GSA was employed in the experiments. The short cavity containing a $10 \mathrm{~mm}$, Brewster-cut, $0.2 \%$ Cr-doped Alexandrite crystal (total pump absorption $=93 \%$ at $532 \mathrm{~nm}$ ) was end pumped with a singlemode, $5 \mathrm{~W}, 532 \mathrm{~nm}$ pump laser. During the experiments, the crystal was kept inside a copper holder and maintained near $20^{\circ} \mathrm{C}$ by water cooling. The end high reflector M6 was later removed, and the overall length of the resonator was increased with a notched, MPC. In order to make the MPC $q$-preserving, the curved (M7, $R=2 \mathrm{~m})$ and the flat (M8) high reflectors were separated by a distance of $114 \mathrm{~cm}$, and the end high reflector (M12, $R=1 \mathrm{~m}$ ) was positioned at a distance of $114 \mathrm{~cm}$ from M8. The design rules of this class of notched, $q$-preserving MPCs were discussed in earlier studies [36]. With the MPC, an additional optical path length of $50.2 \mathrm{~m}$ was added to the Alexandrite resonator, giving a pulse repetition frequency of $5.56 \mathrm{MHz}$.

The continuous-wave power performance of the Alexandrite laser was investigated for the short and extended resonators. Figure 2(a) shows the measured power efficiency of the $x$-cavity Alexandrite laser by using four output couplers with $0.1 \%$, $0.25 \%, 0.5 \%$, and $1.7 \%$ transmission, respectively, near $750 \mathrm{~nm}$. The resulting measured slope efficiencies with four different output couplers were $2.8 \%, 5.9 \%, 11.4 \%$, and $13.9 \%$, respectively. The round trip passive loss of the $x$-cavity Alexandrite laser was estimated to be $0.61 \%$ by using the threshold pump power data shown in Fig. 2(b).

Figure 3 shows the continuous-wave power efficiency data of the short-cavity and extended-cavity Alexandrite laser operating with the $0.25 \%$ output coupler. The MPC extension decreased the power slope efficiency of the $x$-cavity Alexandrite laser from $5.9 \%$ to $1.7 \%$. The insertion of the graphene sample further decreased the slope efficiency of the laser to $0.7 \%$. The round trip passive loss introduced by the MPC and the GSA was estimated to be $3.05 \%$ and $4.82 \%$, respectively, based on the threshold data in Fig. 3.
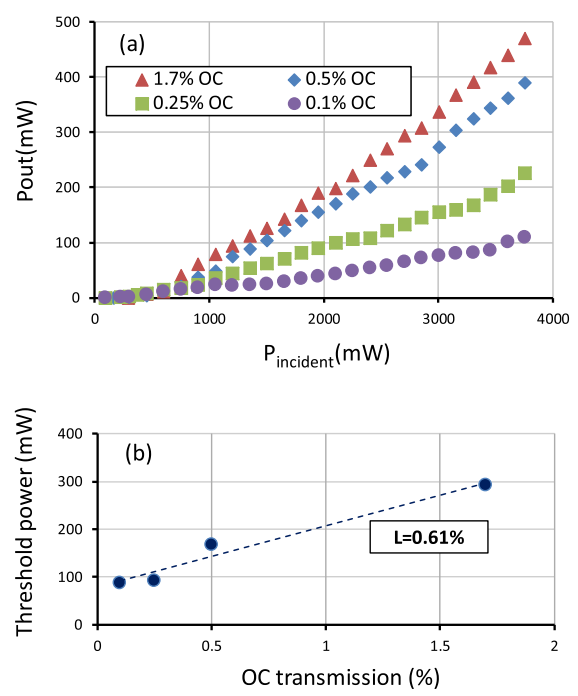

Fig. 2. (a) Continuous-wave power efficiency curves of the $x$-cavity Alexandrite laser measured with four output couplers. (b) Measured threshold pump power as a function of output coupler transmission.

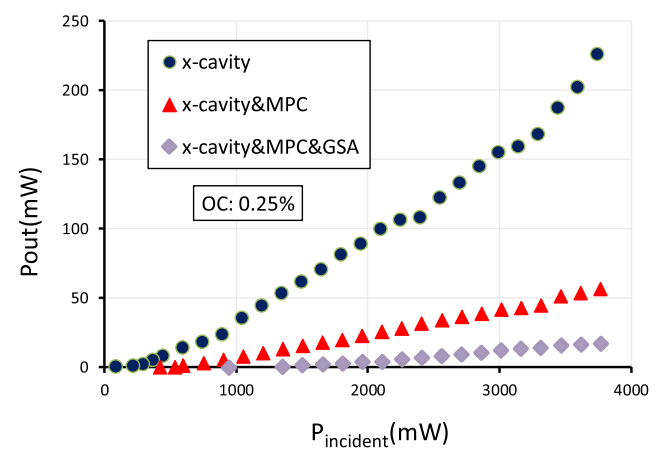

Fig. 3. Measured power efficiency curves of the short cavity, multipass cavity, and the composite cavity containing the MPC and the graphene saturable absorber. The output coupler had a transmission of $0.25 \%$.

In the mode-locking experiments described here, it is important to ensure that pulse generation is indeed initiated via the saturable absorber action in graphene and not by KLM. To investigate the cavity configurations for graphene mode locking and KLM, the separation (d in Fig. 1) between the curved mirrors M1 and M2 was varied at $10 \mu \mathrm{m}$ steps, and the output power of the laser was measured across the full stability range of the resonator. At each M1-M2 separation, the output coupler was translated to see whether mode locking could be initiated. Two separate cases were investigated. In the first case, GSA transferred onto an infrasil substrate was located at the waist position between M13 and M14, and the output power was measured as a function of $d$ at the input pump power of $5 \mathrm{~W}$ [Fig. 4(a)]. The separations where mode locking could be initiated are marked with solid red triangles in Fig. 4(a). Note that when GSA was used, mode locking could be initiated nearly across the full stability range. In the second case, a bare infrasil substrate without graphene was placed between M13 

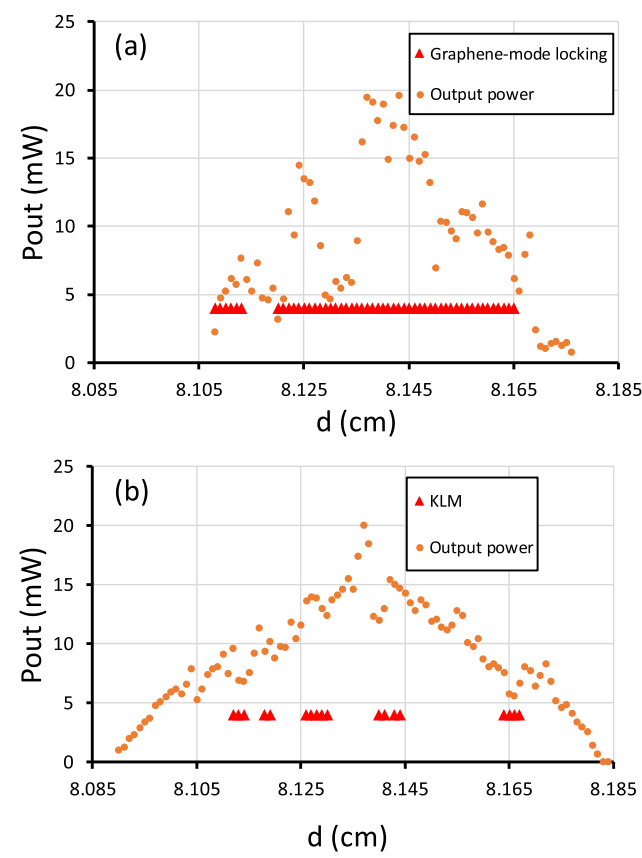

Fig. 4. Measured output power and mode-locking tendency (solid triangles) of the Alexandrite laser as a function of the M2-M3 separation position for (a) GSA and (b) bare infrasil substrate between M13 and M14.

and M14. To keep the output power and, hence, the intracavity pulse energy at about the same level as in the first case, the input pump power was reduced to $2.7 \mathrm{~W}$. Here, the modelocking initiation mechanism is Kerr lensing. Note that KLM action could be observed at fewer points due to the requirement for critical cavity alignment to initiate Kerr lensing [Fig. 4(b)]. Based on the data shown in Figs. 4(a) and 4(b), graphene mode-locking data of the Alexandrite laser were recorded near $d=8.161 \mathrm{~cm}$, where KLM action was not observed with bare infrasil substrate. In the mode-locking experiments, negative group delay dispersion (GDD) was provided by most of the cavity mirrors, including the MPC mirrors. The dispersion control mirrors M4, M5, M7, M8, M9, and M12 each had a GDD of $-50 \mathrm{fs}^{2}$ per bounce, whereas M10 and M11 had a GDD of $-100 \mathrm{fs}^{2}$, and M3 had a GDD of $-40 \mathrm{fs}^{2}$ per bounce. By taking into account the GDD due to the gain medium $\left(60 \mathrm{fs}^{2}\right.$ per $\mathrm{mm}$ [37]), air $\left(20 \mathrm{fs}^{2}\right.$ per meter), the infrasil substrate $\left(\sim 100 \mathrm{fs}^{2}\right.$ per $\left.1 \mathrm{~mm}\right)$, the mirrors M1, M2, and OC ( $\sim 5 \mathrm{fs}^{2}$ per bounce), as well as the mirrors M13-M14 ( 25 fs ${ }^{2}$ per bounce), the net round trip GDD of the composite resonator shown in Fig. 1 was estimated to be around $-400 \mathrm{fs}^{2}$ near $750 \mathrm{~nm}$.

Figures 5 and 6 show the mode-locking results obtained from the MPC Alexandrite laser. Figure 5(a) shows the interferometric autocorrelation trace of the graphene mode-locked Alexandrite laser. The temporal duration of the pulses was determined to be $65 \mathrm{fs}$, by assuming a sech ${ }^{2}$ intensity profile. Figure 5(b) further shows the measured optical spectrum of the generated pulses and the estimated round trip GDD of the resonator as a function of wavelength. The spectral width (full width at half-maximum) was measured to be $9.3 \mathrm{~nm}$. The measured time-bandwidth product of 0.319 suggests that the
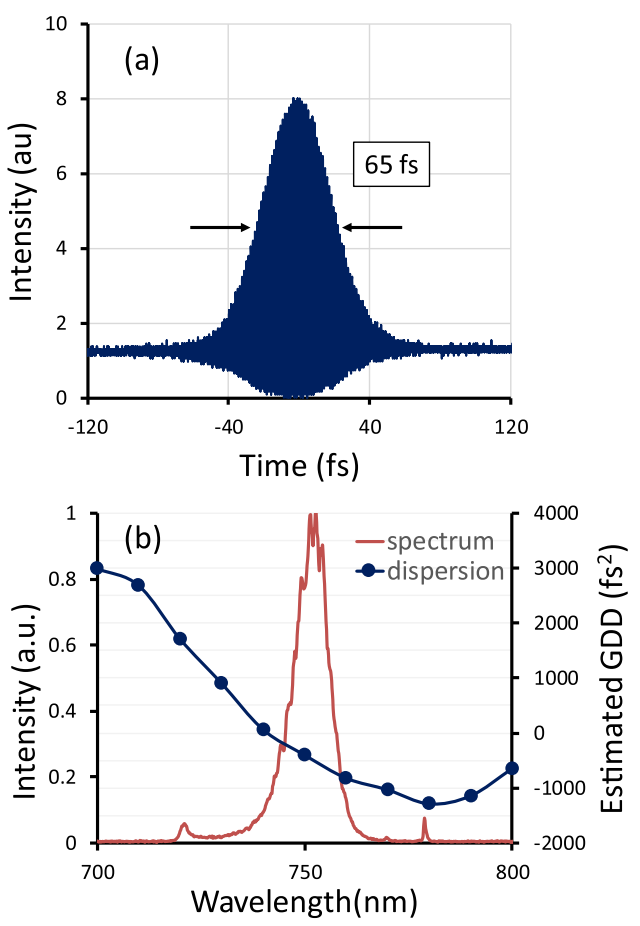

Fig. 5. (a) Interferometric autocorrelation trace of the $65 \mathrm{fs}$ pulses obtained from the graphene mode-locked Alexandrite laser. (b) Measured optical spectrum of the mode-locked pulses and the estimated group delay dispersion as a function of wavelength.
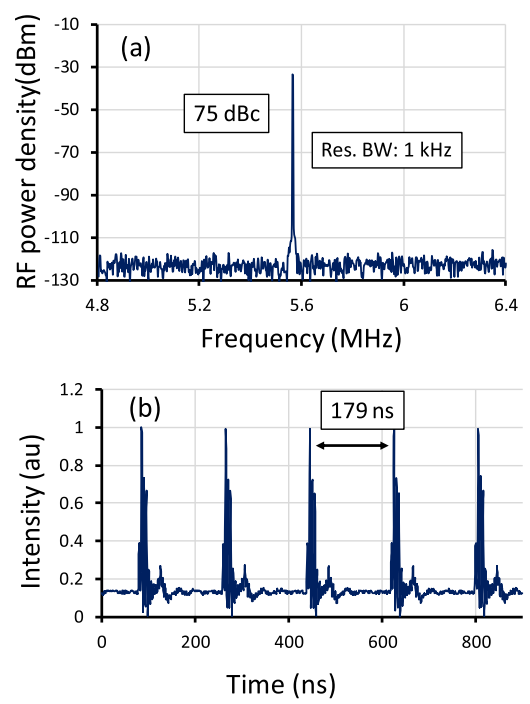

Fig. 6. Measured (a) radio frequency (RF) spectrum and (b) pulse train of the pulses generated with the graphene mode-locked Alexandrite laser at the repetition frequency of $5.56 \mathrm{MHz}$. In the RF spectrum measurements, the resolution bandwidth (Res. BW) was $1 \mathrm{kHz}$.

pulses were nearly transform limited. Note that the net group delay dispersion of the resonator was positive at wavelengths below $740 \mathrm{~nm}$, preventing solitary pulse generation with spectra that span the full tuning range of the Alexandrite laser. 
We foresee that by using cavity optics with optimized GDD over the entire tuning range, it should be possible to generate sub-10 fs pulses from the Alexandrite laser [15]. At the pump power of $5 \mathrm{~W}$, the output power of the laser during modelocked operation was $8 \mathrm{~mW}$, corresponding to $1.4 \mathrm{~nJ}$ of pulse energy at the pulse repetition frequency of $5.56 \mathrm{MHz}$. The peak power was $22 \mathrm{~kW}$. Based on the ABCD analysis of the cavity, the spot size on the GSA was estimated to be $25 \mu \mathrm{m}$, yielding an incident fluence of $20 \mathrm{~mJ} / \mathrm{cm}^{2}$ on the GSA. Figures 6(a) and 6(b) further show the measured radio frequency (RF) spectrum and the pulse train of the graphene mode-locked MPC Alexandrite laser. As can be seen from Fig. 6(a), the pulse train carrier signal at $5.56 \mathrm{MHz}$ had a $75 \mathrm{dBc}$ contrast with respect to the noise background, indicating mode-locked operation without $Q$-switching instabilities.

In conclusion, we report for the first time, to the best our knowledge, graphene mode-locked operation of a femtosecond Alexandrite laser. By using a MPC architecture and with $5 \mathrm{~W}$ of pump power at $532 \mathrm{~nm}$, we obtained $65 \mathrm{fs}$, nearly transformlimited pulses at $750 \mathrm{~nm}$. The RF peak at the repetition frequency of $5.56 \mathrm{MHz}$ had a contrast of $75 \mathrm{dBc}$ with respect to the background noise level. The energy, peak power, and the time-bandwidth product of the generated pulses were $1.4 \mathrm{~nJ}$, $22 \mathrm{~kW}$, and 0.319 , respectively. Finally, this represents the shortest wavelength, to our knowledge, where graphene has been utilized to initiate mode-locked operation of a laser.

Acknowledgment. We thank Abdullah Muti, Ferda Canbaz, and Ișınsu Baylam for their help during the experiment.

\section{REFERENCES}

1. J. M. Dawlaty, S. Shivaraman, M. Chandrashekhar, F. Rana, and M. G. Spencer, Appl. Phys. Lett. 92, 042116 (2008).

2. Q. Bao, H. Zhang, Y. Wang, Z. Ni, Y. Yan, Z. X. Shen, K. P. Loh, and D. Y. Tang, Adv. Funct. Mater. 19, 3077 (2009).

3. Y. Fujimoto, T. Suzuki, R. A. M. Ochante, T. Hirayama, M. Murakami, H. Shiraga, M. Yoshida, O. Ishii, and M. Yamazaki, Electron. Lett. 50, 1470 (2014).

4. S. Kajikawa, M. Yoshida, O. Ishii, M. Yamazaki, and Y. Fujimoto, Opt. Commun. 424, 13 (2018).

5. I. Baylam, S. Ozharar, N. Kakenov, C. Kocabas, and A. Sennaroglu, Opt. Lett. 42, 1404 (2017).

6. I. H. Baek, H. W. Lee, S. Bae, B. H. Hong, Y. H. Ahn, D.-I. Yeom, and F. Rotermund, Appl. Phys. Express 5, 032701 (2012).

7. F. Canbaz, N. Kakenov, C. Kocabas, U. Demırbas, and A. Sennaroglu, Opt. Express 25, 2834 (2017).

8. S. Davide Di Dio Cafiso, E. Ugolotti, A. Schmidt, V. Petrov, U. Griebner, A. Agnesi, W. B. Cho, B. H. Jung, F. Rotermund, S. Bae, B. H. Hong, G. Reali, and F. Pirzio, Opt. Lett. 38, 1745 (2013).

9. M. N. Cizmeciyan, J. W. Kim, S. Bae, B. H. Hong, F. Rotermund, and A. Sennaroglu, Opt. Lett. 38, 341 (2013).
10. G. Zhu, X. Zhu, F. Wang, S. Xu, Y. Li, X. Guo, K. Balakrishnan, R. A. Norwood, and N. Peyghambarian, IEEE Photon. Technol. Lett. 28, 7 (2016).

11. G. Zhu, X. Zhu, K. Balakrishnan, R. A. Norwood, and N. Peyghambarian, Opt. Mater. Express 3, 1365 (2013).

12. M. J. Damzen, G. M. Thomas, and A. Minassian, Opt. Express 25, 11622 (2017).

13. U. Parali, X. Sheng, A. Minassian, G. Tawy, J. Sathian, G. M. Thomas, and M. J. Damzen, Opt. Commun. 410, 970 (2018).

14. E. Beyatli, I. Baali, B. Sumpf, G. Erbert, A. Leitenstorfer, A. Sennaroglu, and U. Demirbas, J. Opt. Soc. Am. B 30, 3184 (2013).

15. I. Yorulmaz, E. Beyatli, A. Kurt, A. Sennaroglu, and U. Demirbas, Opt. Mater. Express 4, 776 (2014).

16. M. Fibrich, J. Šulc, D. Vyhlídal, H. Jelínková, and M. Čech, Laser Phys. 27, 115801 (2017).

17. A. Munk, B. Jungbluth, M. Strotkamp, H. D. Hoffmann, R. Poprawe, J. Höffner, and F. J. Lübken, Opt. Express 26, 14928 (2018).

18. S. Ghanbari, R. Akbari, and A. Major, Opt. Express 24, 14836 (2016).

19. S. Ghanbari, K. A. Fedorova, A. B. Krysa, E. U. Rafailov, and A. Major, Opt. Lett. 43, 232 (2018)

20. C. Cihan, A. Muti, I. Baylam, A. Kocabas, U. Demirbas, and A. Sennaroglu, Opt. Lett. 43, 1315 (2018).

21. Y. Zhong, Z. Cai, D. Wu, Y. Cheng, J. Peng, J. Weng, Z. Luo, B. Xu, and H. Xu, IEEE Photon. Technol. Lett. 28, 1755 (2016).

22. M. Gaponenko, P. W. Metz, A. Härkönen, A. Heuer, T. Leinonen, M. Guina, T. Südmeyer, G. Huber, and C. Kränkel, Opt. Lett. 39, 6939 (2014).

23. W. Li, T. Du, J. Lan, C. Guo, Y. Cheng, H. Xu, C. Zhu, F. Wang, Z. Luo, and Z. Cai, Opt. Lett. 42, 671 (2017).

24. B. Xu, S. Luo, X. Yan, J. Li, J. Lan, Z. Luo, H. Xu, Z. Cai, H. Dong, J. Wang, and L. Zhang, IEEE J. Sel. Top. Quantum Electron. 23, 1900507 (2017).

25. V. G. Savitski, P. J. Schlosser, J. E. Hastie, A. B. Krysa, J. S. Roberts, M. D. Dawson, D. Burns, and S. Calvez, IEEE Photon. Technol. Lett. 22, 209 (2010).

26. H.-Y. Lin, W.-S. Li, J.-L. Lan, X.-F. Guan, H.-Y. Xu, and Z.-P. Cai, Appl. Opt. 56, 802 (2017).

27. W. Li, J. Peng, Y. Zhong, D. Wu, H. Lin, Y. Cheng, Z. Luo, J. Weng, H. Xu, and Z. Cai, Opt. Mater. Express 6, 2031 (2016).

28. D. Wu, Z. Cai, Y. Zhong, J. Peng, Y. Cheng, J. Weng, Z. Luo, and H. Xu, IEEE J. Sel. Top. Quantum Electron. 23, 7 (2017).

29. B. Chen, X. Zhang, K. Wu, H. Wang, J. Wang, and J. Chen, Opt. Express 23, 26723 (2015).

30. J. Sotor, G. Sobon, and K. M. Abramski, Opt. Express 22, 13244 (2014).

31. J. Sotor, G. Sobon, M. Kowalczyk, W. Macherzynski, P. Paletko, and K. M. Abramski, Opt. Lett. 40, 3885 (2015).

32. F. Zhang, S. Han, Y. Liu, Z. Wang, and X. Xu, Appl. Phys Lett. 106, 091102 (2015).

33. A. Marini, J. D. Cox, and F. J. García de Abajo, Phys. Rev. B 95 125408 (2017).

34. W. Gadomski and B. Ratajska-Gadomska, J. Opt. Soc. Am. B 17, 188 (2000).

35. F. Canbaz, N. Kakenov, C. Kocabas, U. Demirbas, and A. Sennaroglu, Opt. Lett. 40, 4110 (2015).

36. A. Sennaroglu, A. M. Kowalevicz, E. P. Ippen, and J. G. Fujimoto, IEEE J. Quantum Electron. 40, 519 (2004).

37. P. Loiko and A. Major, Opt. Mater. Express 6, 2177 (2016). 